Orbett T. Alexander*, Alice Brink and Hendrik G. Visser

\title{
The crystal structure of bis $\left(\mu_{2}-5,7-\right.$ dichloroquinolin-8-olato- $\left.\kappa^{3} N, 0: 0\right)$ - tetrakis(5,7-dichloroquinolin-8-olato- $\mathrm{K}^{2} \mathrm{~N}, \mathrm{O}$ ) bis (methanol- $\mathrm{K}^{1} \mathrm{O}$ ) dieuropium(III) - toluene (1/1), $\mathrm{C}_{63} \mathrm{H}_{39} \mathrm{Cl}_{12} \mathrm{Eu}_{2} \mathrm{~N}_{6} \mathrm{O}_{8}$
}

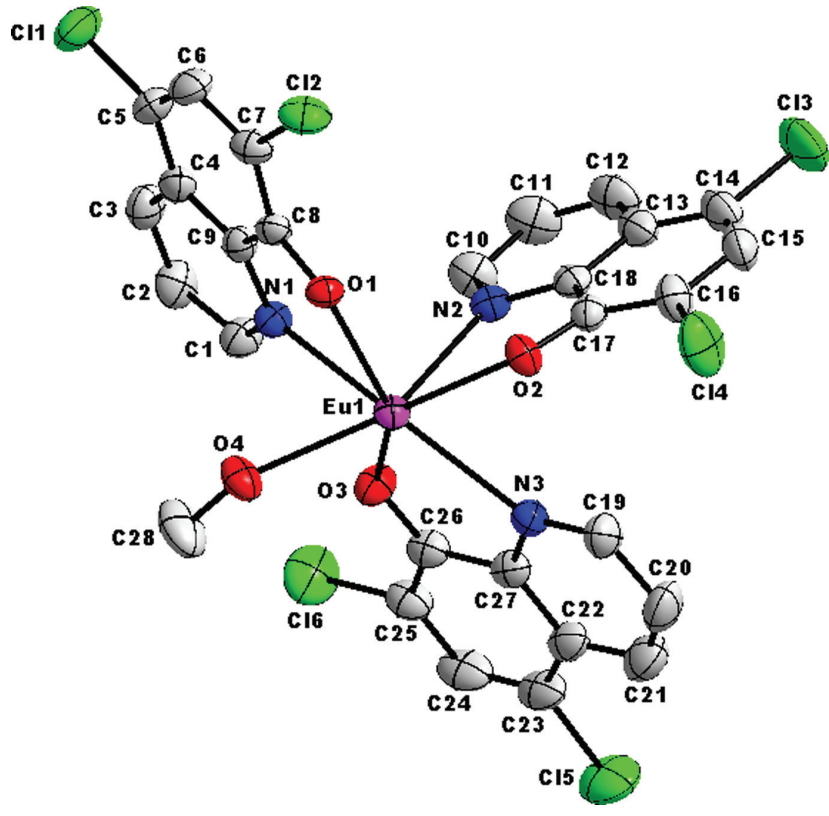

https://doi.org/10.1515/ncrs-2019-0606

Received August 21, 2019; accepted October 2, 2019; available online October 25, 2019

\section{Abstract \\ $\mathrm{C}_{63} \mathrm{H}_{39} \mathrm{Cl}_{12} \mathrm{Eu}_{2} \mathrm{~N}_{6} \mathrm{O}_{8}$, triclinic, $P \overline{1}$ (no. 2), $a=10.720(5) \AA$, $b=12.232(5) \AA, \quad c=14.267(5) \AA, \quad \alpha=65.288(32)^{\circ}$, $\beta=71.325(5)^{\circ}, \quad \gamma=88.067(5)^{\circ}, \quad V=1599.1(11) \AA 33, \quad Z=1$, $R_{g t}(F)=0.0358, w R_{\text {ref }}\left(F^{2}\right)=0.0785, T=293(2) \mathrm{K}$.}

\section{CCDC no.: 1913793}

The asymmetric unit of the dinuclear title crystal structure is shown in the figure. Table 1 contains crystallographic data and Table 2 contains the list of the atoms including atomic coordinates and displacement parameters.

*Corresponding author: Orbett T. Alexander, Department of Chemistry, University of the Free State, Bloemfontein 9301, South Africa, e-mail: alexanderorbett@gmail.com. https://orcid.org/0000-0003-4926-8342

Alice Brink and Hendrik G. Visser: Department of Chemistry, University of the Free State, Bloemfontein 9301, South Africa
Table 1: Data collection and handling.

\begin{tabular}{ll}
\hline Crystal: & Yellow block \\
Size: & $0.22 \times 0.19 \times 0.12 \mathrm{~mm}$ \\
Wavelength: & Mo $K \alpha$ radiation $(0.71073 \AA$ A) \\
$\mu:$ & $2.51 \mathrm{~mm}^{-1}$ \\
Diffractometer, scan mode: & Bruker APEX-II, $\varphi$ and $\omega$-scans \\
$\theta_{\text {max }}$, completeness: & $28^{\circ},>99 \%$ \\
$N(h k l)_{\text {measured }}, N(h k l)_{\text {unique }}, R_{\text {int }}:$ & $34241,7633,0.077$ \\
Criterion for $I_{\text {obs }}, N(h k l)_{\mathrm{gt}}:$ & $I_{\text {obs }}>2 \sigma\left(I_{\text {obs }}\right), 6291$ \\
$N(\text { param })_{\text {refined }}:$ & 48 \\
Programs: & Bruker programs [1], SIR97 [2], \\
& OLEX2 [3], SHELX [4], \\
& DIAMOND [5] \\
\hline
\end{tabular}

\section{Source of materials}

In this two-step synthesis, [tris-(5,7-dichloro-8-hydroxyquinoline $\left.)\left(\mathrm{H}_{2} \mathrm{O}\right)_{2} \mathrm{Eu}(\mathrm{III})\right]$ was prepared first by dissolving 5,7dichloro-8-hydroxyquinoline $(0.707 \mathrm{~g}, 3.33 \mathrm{mmol})$ in $15 \mathrm{~mL}$ of absolute ethanol and then reacting with $\mathrm{EuCl}_{3} \cdot 6 \mathrm{H}_{2} \mathrm{O}$ $(0.308 \mathrm{~g}, 0.84 \mathrm{mmol})$ in $20 \mathrm{~mL}$ of distilled water. $\mathrm{NaOH}$ $(0.266 \mathrm{~g}, 6.5 \mathrm{mmol})$ in $10 \mathrm{~mL}$ of distilled water was added after one hour. Yellow-orange cubic crystals were obtained from ethanol solvent after one week. Yield: $0.4747 \mathrm{~g}, 68 \%$. UV-Vis $\left(\mathrm{nm} ; \mathrm{L} \mathrm{mol}^{-1} \mathrm{~cm}^{-1}\right): \lambda_{\max }=408, \epsilon=5 \mathrm{E}-3$.

In the second reaction step [(5,7-dichloro-8hydroxyquinoline) $\left.\left(\mathrm{H}_{2} \mathrm{O}\right)_{2} \mathrm{Eu}(\mathrm{III})\right](1 \mathrm{mg}, 1.21 \mathrm{mmol})$ in $20 \mathrm{~mL}$ of toluene was refluxed with 2,2'-bipyridine $(1.9 \mathrm{mg})$ for six hours with vigorous stirring using a Dean Stark setup to remove excess water. The colour of the solution turned yelloworange after a while. The reaction solution was filtered and layered with methanol and then left to crystallize. Yellowish crystals precipitated within a few hours.

\section{Experimental details}

The aromatic $\mathrm{H}$ atoms were placed in geometrically idealized positions and constrained to ride on their parent atoms, with $\mathrm{C}-\mathrm{H}=0.95$ and $0.98 \AA$ and $U_{\text {iso }}(\mathrm{H})=1.5 U_{\text {eq }}(\mathrm{C})$ and 1.2 $U_{\text {eq }}(\mathrm{C})$, respectively. The placement of the $\mathrm{H}$ atoms of the methyl group was that of an idealised methyl group according to the electron-density map (HFIX 137). The highest peak is 0.87 e. $\AA^{-3}$ and deepest hole is -0.69 e. $\AA^{-3}$. 
Table 2: Fractional atomic coordinates and isotropic or equivalent isotropic displacement parameters $\left(\AA^{2}\right)$.

\begin{tabular}{|c|c|c|c|c|}
\hline Atom & $x$ & $y$ & $z$ & $U_{\text {iso }} * / U_{\text {eq }}$ \\
\hline Eu1 & $0.54445(2)$ & $0.54657(2)$ & $0.34215(2)$ & $0.02670(6)$ \\
\hline 01 & $0.3651(2)$ & $0.4942(2)$ & $0.51137(16)$ & $0.0282(5)$ \\
\hline 02 & $0.5502(2)$ & $0.7324(2)$ & $0.35541(16)$ & $0.0329(6)$ \\
\hline 03 & $0.6046(2)$ & $0.5072(2)$ & $0.19228(17)$ & $0.0368(6)$ \\
\hline 04 & $0.5610(3)$ & $0.3291(2)$ & $0.42868(19)$ & $0.0416(7)$ \\
\hline $\mathrm{H} 4$ & $0.524(4)$ & $0.304(4)$ & $0.491(3)$ & 0.062 * \\
\hline N1 & $0.3384(3)$ & $0.4253(2)$ & $0.3613(2)$ & $0.0307(7)$ \\
\hline N2 & $0.4262(3)$ & $0.7128(3)$ & $0.2262(2)$ & $0.0318(7)$ \\
\hline N3 & $0.7478(3)$ & $0.6933(3)$ & $0.1754(2)$ & $0.0338(7)$ \\
\hline $\mathrm{Cl} 1$ & $-0.15406(11)$ & $0.26395(11)$ & $0.58949(9)$ & $0.0602(3)$ \\
\hline $\mathrm{Cl} 2$ & $0.13815(10)$ & $0.52058(10)$ & $0.68640(7)$ & $0.0450(2)$ \\
\hline $\mathrm{Cl} 3$ & $0.36730(15)$ & $1.17002(11)$ & $0.07931(10)$ & $0.0748(4)$ \\
\hline $\mathrm{Cl} 4$ & $0.64713(14)$ & $0.96320(10)$ & $0.34746(10)$ & $0.0686(4)$ \\
\hline $\mathrm{Cl} 5$ & $1.09450(13)$ & $0.72247(13)$ & $-0.18544(9)$ & $0.0800(4)$ \\
\hline $\mathrm{Cl} 6$ & $0.65696(14)$ & $0.40191(12)$ & $0.03112(9)$ & $0.0680(4)$ \\
\hline $\mathrm{C} 1$ & $0.3285(4)$ & $0.3817(3)$ & $0.2935(3)$ & $0.0394(9)$ \\
\hline $\mathrm{H} 1$ & 0.4022 & 0.3955 & 0.2319 & $0.047^{\star}$ \\
\hline $\mathrm{C} 2$ & $0.2139(4)$ & $0.3156(3)$ & $0.3083(3)$ & $0.0431(10)$ \\
\hline $\mathrm{H} 2$ & 0.2135 & 0.2838 & 0.2595 & $0.052^{\star}$ \\
\hline C3 & $0.1024(4)$ & $0.2988(3)$ & $0.3961(3)$ & $0.0418(9)$ \\
\hline H3 & 0.0242 & 0.2579 & 0.4060 & $0.050^{\star}$ \\
\hline $\mathrm{C} 4$ & $0.1076(4)$ & $0.3439(3)$ & $0.4708(3)$ & $0.0346(8)$ \\
\hline $\mathrm{C5}$ & $-0.0011(4)$ & $0.3351(3)$ & $0.5631(3)$ & $0.0384(9)$ \\
\hline C6 & $0.0103(4)$ & $0.3869(4)$ & $0.6278(3)$ & $0.0425(10)$ \\
\hline H6 & -0.0634 & 0.3833 & 0.6859 & 0.051 * \\
\hline $\mathrm{C} 7$ & $0.1316(4)$ & $0.4455(3)$ & $0.6079(3)$ & $0.0346(8)$ \\
\hline $\mathrm{C} 8$ & $0.2464(3)$ & $0.4491(3)$ & $0.5259(2)$ & $0.0279(7)$ \\
\hline C9 & $0.2289(3)$ & $0.4040(3)$ & $0.4528(2)$ & $0.0299(8)$ \\
\hline C10 & $0.3607(4)$ & $0.7026(4)$ & $0.1661(3)$ & $0.0443(10)$ \\
\hline $\mathrm{H} 10$ & 0.3495 & 0.6274 & 0.1664 & $0.053^{\star}$ \\
\hline C11 & $0.3064(5)$ & $0.8003(4)$ & $0.1011(3)$ & $0.0531(11)$ \\
\hline H11 & 0.2582 & 0.7879 & 0.0619 & $0.064^{*}$ \\
\hline C12 & $0.3248(5)$ & $0.9111(4)$ & $0.0960(3)$ & $0.0521(12)$ \\
\hline H12 & 0.2897 & 0.9757 & 0.0530 & $0.063^{*}$ \\
\hline C14 & $0.4269(5)$ & $1.0404(4)$ & $0.1564(3)$ & $0.0498(12)$ \\
\hline C15 & $0.5023(5)$ & $1.0501(4)$ & $0.2126(3)$ & $0.0494(11)$ \\
\hline H15 & 0.5241 & 1.1254 & 0.2083 & $0.059^{\star}$ \\
\hline C16 & $0.5484(4)$ & $0.9464(3)$ & $0.2780(3)$ & $0.0422(10)$ \\
\hline C17 & $0.5171(4)$ & $0.8321(3)$ & $0.2890(3)$ & $0.0319(8)$ \\
\hline C18 & $0.4457(4)$ & $0.8247(3)$ & $0.2225(2)$ & $0.0321(8)$ \\
\hline C13 & $0.3973(4)$ & $0.9286(3)$ & $0.1561(3)$ & $0.0398(10)$ \\
\hline C19 & $0.8195(4)$ & $0.7810(3)$ & $0.1714(3)$ & $0.0434(10)$ \\
\hline H19 & 0.7894 & 0.8071 & 0.2266 & $0.052^{\star}$ \\
\hline $\mathrm{C} 20$ & $0.9408(4)$ & $0.8372(4)$ & $0.0861(4)$ & $0.0570(12)$ \\
\hline $\mathrm{H} 2 \mathrm{O}$ & 0.9899 & 0.8984 & 0.0861 & $0.068^{\star}$ \\
\hline $\mathrm{C} 21$ & $0.9855(4)$ & $0.8016(4)$ & $0.0040(3)$ & $0.0546(12)$ \\
\hline $\mathrm{H} 21$ & 1.0652 & 0.8389 & -0.0524 & $0.066^{\star}$ \\
\hline $\mathrm{C} 22$ & $0.9123(4)$ & $0.7089(4)$ & $0.0038(3)$ & $0.0404(9)$ \\
\hline $\mathrm{C} 23$ & $0.9473(4)$ & $0.6618(4)$ & $-0.0747(3)$ & $0.0486(12)$ \\
\hline C24 & $0.8709(4)$ & $0.5702(4)$ & $-0.0654(3)$ & $0.0509(12)$ \\
\hline $\mathrm{H} 24$ & 0.8968 & 0.5410 & -0.1186 & $0.061^{\star}$ \\
\hline $\mathrm{C} 25$ & $0.7539(4)$ & $0.5186(4)$ & $0.0227(3)$ & $0.0416(10)$ \\
\hline $\mathrm{C} 26$ & $0.7110(4)$ & $0.5569(3)$ & $0.1060(2)$ & $0.0337(8)$ \\
\hline $\mathrm{C} 27$ & $0.7909(4)$ & $0.6556(3)$ & $0.0940(2)$ & $0.0331(8)$ \\
\hline $\mathrm{C} 28$ & $0.5864(6)$ & $0.2385(4)$ & $0.3909(4)$ & $0.0694(16)$ \\
\hline
\end{tabular}

Table 2 (continued)

\begin{tabular}{|c|c|c|c|c|}
\hline Atom & $x$ & $y$ & $z$ & $\boldsymbol{U}_{\text {iso }}{ }^{*} / \boldsymbol{U}_{\mathrm{eq}}$ \\
\hline $\mathrm{H} 28 \mathrm{~A}$ & 0.6223 & 0.2756 & 0.3123 & $0.104^{*}$ \\
\hline $\mathrm{H} 28 \mathrm{~B}$ & 0.5053 & 0.1883 & 0.4146 & $0.104^{\star}$ \\
\hline $\mathrm{H} 28 \mathrm{C}$ & 0.6490 & 0.1899 & 0.4201 & $0.104^{*}$ \\
\hline $\mathrm{C} 29^{\mathrm{a}}$ & $-0.034(2)$ & $0.0017(17)$ & $0.7046(13)$ & $0.173(8)$ \\
\hline $\mathrm{H} 29 \mathrm{~A}^{\mathrm{a}}$ & -0.1262 & -0.0185 & 0.7500 & 0.259 * \\
\hline$H 29 B^{a}$ & -0.0058 & 0.0834 & 0.6874 & $0.259^{*}$ \\
\hline $\mathrm{H} 29 \mathrm{C}^{\mathrm{a}}$ & 0.0177 & -0.0526 & 0.7430 & $0.259^{*}$ \\
\hline C30 & $-0.0176(8)$ & $-0.0096(6)$ & $0.6003(6)$ & $0.1002(17)$ \\
\hline C31 & $0.1062(8)$ & $-0.0082(6)$ & $0.5351(6)$ & $0.1118(17)$ \\
\hline H31 & 0.1763 & -0.0136 & 0.5614 & $0.134^{\star}$ \\
\hline C32 & $0.1315(8)$ & $0.0009(6)$ & $0.4321(6)$ & $0.1178(19)$ \\
\hline H32 & 0.2163 & 0.0015 & 0.3867 & $0.141^{\star}$ \\
\hline
\end{tabular}

\section{Comment}

There are various uses of lanthanides for an array of applications across many scientific spheres. The broader spectrum of applications for this series encapsulates magnetic studies [6, 7], nuclear chemistry [8], catalysis [9, 10], radio-therapy [11, 12], ceramic's $[13,14]$ and many others. Europium ions are extensively utilised in photoluminescence research, particularly as a potential photo-emissive layer in the edifice of optoelectronic devices [15-17]. Owing to its luminescent nature [18], the europium ion conjugated to bulky multi-dentate organic matrices, which are also used as bioimaging agents in biomedical sciences [19, 20]. The use of organic matrices coordinated to the europium ion is primarily to induce photo-sensitization since the metal ion itself exhibits slow emission rates and low absorption coefficients [21]. The whole phenomenon of using organic chromophores to sensitize the europium ion is affectionately known as the antenna effect [22, 23]. This has certainly become a trend of coordinating ligands on the immediate outer shell of the metal ion to access its metallic effect and/or characteristics with respect to various scientific researches undertaken $[24,25]$.

The dinuclear crystal structure was solved in the centrosymmetric space group, $P \overline{1}$, with only half a complex in the asymmetric unit. It has an inversion centre which propagates right between the two europium metal centres justifying the dimeric nature of the two joint isostructural monomeric halves. This dimeric behaviour was also observed by Y.-C. Liu et al. with zinc [26]. The crystal structure is stabilized by inter- and intra-molecular interactions. The intra-molecular hydrogen interactions occur between the para-substituted chlor substituents ( $\mathrm{Cl} 1, \mathrm{Cl} 3$ and $\mathrm{Cl} 5$ ) with the nearby hydrogen atoms riding on $\mathrm{C} 3, \mathrm{C} 12$ and $\mathrm{C} 21$ respectively. There are three sets of bifurcation networks observed in this crystal structure. The first bifurcation occurs on oxygen $\mathrm{O} 2$ with hydrogen 
atoms riding on oxygen $\mathrm{O} 4$ of the coordinated methanol solvent and carbon $\mathrm{C} 19$ of the pyridyl ring of the ligand respectively making an angle of $128.88^{\circ}$. Furthermore, $\mathrm{Cl} 1$ and $\mathrm{Cl} 5$ undergo $90^{\circ}$ bifurcation with hydrogen atoms riding on $\mathrm{C} 3$, C29 (H29B) and C21, C29 (H29C) respectively. There is half a molecule of the toluene solvent trapped in the asymmetric unit cell, also affected by the inversion centre. Subsequently, the para-substituted carbon of this solvent is disordered (50:50\%) equally over two positions.

Acknowledgements: This work is based on the research supported in part by the National Research Foundation of South Africa (Gran UID: 99139) The authors would also like to thank the University of the Free State for financial support.

\section{References}

1. Bruker, SAINT-Plus (Version 7.12), Bruker AXS Inc., Madison, WI, USA (2004).

2. Altomare, A.; Burla, M. C.; Camalli, M.; Cascarano, G. L.; Giacovazzo, C.; Guagliardi, A.; Moliterni, A. G. G.; Polidori, G.; Spagna, R.: A new tool for crystal structure determination and refinement. J. Appl. Cryst. 32 (1999) 115-119.

3. Dolomanov, O. V.; Bourhis, L. J.; Gildea, R. J.; Howard, J. A. K.; Puschmann, H.: OLEX2: A complete structure solution, refinement and analysis program. J. Appl. Cryst., 42 (2009) 339-341.

4. Sheldrick, G. M.: A short history of SHELX. Acta Crystallogr. Sect. A. A64 (2008) 112-122.

5. Brandenbug, K.; Putz, H.; DIAMOND. Visual crystal structure information system; Version 3.0c Crystal Impact GbR, Bonn, Germany (2005).

6. Akkus, T.; Uğurlu, M.; Demir, L.: Variation of coherent to Compton scattering differential cross-section ratios of some lanthanides with the external magneticfield at $59.54 \mathrm{keV}$. Result Phys. 13 (2019) 1-4.

7. Soek, R. N.; Ferreira, C. M.; Santana, F. S.; Hughes, D. L.; Poneti, G.; Ribeiro, R. R.; Nunes, F. S.: Structure and magnetic properties of two new lanthanide complexes with the 1-( $(E)$ 2-pyridinylmethylidene)semicarbazone ligand. J. Mol. Struct. 1184 (2019) 254-261.

8. Bunzli, J. C. G.: Lanthanide probes in life, Chemical and earth sciences. Elsevier, Netherlands (1989).

9. Shibasaki, M.; Yoshikawa, N.: Lanthanide complexes in multifunctional asymmetric catalysis. Chem. Rev. 102 (6) (2002) 2187-2210.

10. Mikami, K.; Terada, M.; Matsuzawa, H.: Asymmetric catalysis by lanthanide complexes. Angew. Chem., Int. Ed. 41 (2002) 3554-3571.
11. Fricker, S. P.: The therapeutic application of lanthanides. Chem. Soc. Rev. 35 (2006) 524-533.

12. Kostova, I.: Lanthanides as anticancer agents. Curr. Med. Chem. - Anti-Cancer Agents. 5 (2005) 591-602.

13. Bardez-Giboire, I.; Kidari, A.; Magnin, M.; Dussossoy, J.; Peuget, S.; Caraballo, R.; Tribet, M.; Doreau, F.; Jegou, C.: Americium and trivalent Lanthanides incorporation in high-level waste glass-ceramics. J. Nucl. Mater. 492 (2017) 231-238.

14. Crum, J. V.; Turo, L.; Riley, B.; Tang, M.; Kossoy, A.: Multi-phase glass-ceramics as a waste form for combined fission products: Alkalis, alkaline earths, lanthanides, and transition metals. J. Am. Ceram. Soc. 95 (4) (2012) 1297-1303.

15. Eliseeva, S. V.; Bunzli, J. C.: Lanthanide luminescence for functional materials and bio-sciences. Chem. Soc. Rev. 39 (1) (2010) 189-227.

16. Gallardo, H.; Braga, H. C.; Tuzimoto, P.; Bortoluzzi, A.; Salla, C. A. M.; Bechtold, I. H.; Martins, J. S.; Legnani, C.; Quirino, W. G.: Synthesis, structure and OLED application of a new europium(III)complex:\{tris (thenoyltrifluoroacetonate) $[1,2,5]$ selenadiazolo[3,4f][1,10]phenanthroline europium(III). Inorgica Chim. Acta. 473 (2018) 75-82.

17. Biju, S.; Raj, D. B. A.; Reddy, M. L. P.; Kariuki, B. M.: Synthesis, crystal structure, and luminescent properties of novel $\mathrm{Eu}^{3+}$ heterocyclic $\beta$-diketonate complexes with bidentate nitrogen donors. Inorg. Chem. 45 (2006) 10651-10660.

18. Werts, M. H. V.: Making sense of lanthanide luminescence. Sci. Progress. 88 (2) (2005) 101-131.

19. Amoroso, A. J.; Pope, S. J.: Using lanthanide ions in molecular bio-imaging. Chem. Soc. Rev. 44 (14) (2015) 4723-4742.

20. Bunzli, J. C.; Piguet, C.: Taking advantage of luminescent lanthanide ions. Chem. Soc. rev. 34 (12) (2005) 1048-1077.

21. Im, S. Y.; Go, D. H.; Ryu, J. G.; Kim, Y. S.: New Narrow-Band Luminescence Using Lanthanide Coordination Compounds for Light-Emitting Diodes. IEICE Trans. Electronics. E100.C (11) (2017) 1021-1025.

22. Bünzli, J. C. G.: On the design of highly luminescent lanthanide complexes. Coord. Chem. Rev. 293-294 (2015) 19-47.

23. Alpha, B.; Ballardini, R.; Balzani, V.; Lehn, J. M.; Perathoner, S.; Sabbatini, N.: Antenna effect in luminescent lanthanide cryptates: a photophysical study. Photochem. Photobiol. 52 (2) (1990) 299-306.

24. Suli, L. M.; Ibrahim, W. H. W.; Aziz, B. A.; Deraman, M. R.; Ismail, N. A.: A review of rare earth mineral processing technology, Chem. Eng. Res. Bull. 19 (2013) 20-35.

25. Bünzli, J. C. G.; Eliseeva, S. V.: Photophysics of lanthanoid coordination compounds. Elsevier (2013) 339-398.

26. Liu, Y.-C.; Wei, J.-H.; Chen, Z.-F.; Liu, M.; Gu, Y.-Q.; Huang, K.-B.; Li, Z.-Q.; Liang, H.: The antitumor activity of zinc(II) and copper(II) complexes with 5,7-dihalo-substituted-8-quinolinoline. Eur. J. Med. Chem. 69 (2013) 554-563. 